\title{
Detection of Carotid Artery Stenosis with Intraplaque Hemorrhage and Neovascularization with a Scanning Interferometer
}

\author{
Raiyan Tripti Zaman ${ }^{1,2,3,4^{*}}$, Hisanori Kosuge ${ }^{3 \#}$, Sanjiv Sam Gambhir ${ }^{5+,}$, Lei Xing ${ }^{4}$
}

1. Department of Radiology, Harvard Medical School, Boston, MA-02129, USA

2. Gordon Center for Medical Imaging, Massachusetts General Hospital, Boston, MA-02129, USA

3. Department of Medicine, Division of Cardiovascular Medicine, Stanford University School of Medicine, Stanford, CA-94305, USA

4. Division of Medical Physics, Department of Radiation Oncology, Stanford University School of Medicine, Stanford, CA-94305, USA

5. Department of Radiology, Stanford University School of Medicine, Stanford, CA-94304, USA

\#Current affiliation: Department of Cardiology, Tokyo Medical University, 6-7-1 Nishishinijuku, Shinjuku-ku, Tokyo 160-0023, Japan

†Deceased (1962-2020)

${ }^{*}$ Corresponding author's current affiliation:

Raiyan T. Zaman, Ph.D., MSEE

Assistant Professor, Department of Radiology

Harvard Medical School

Assistant Investigator, Gordon Center for Medical Imaging

Massachusetts General Hospital

149, 13th Street, Charlestown, Boston, MA 02129

Tel: (617) 643-9610 (O); (512) 294-7327 (C)

Email: rzaman@mgh.harvard.edu 


\section{SUPPLEMENTAL SECTIONS}

\section{Excitation System}

A nanosecond tunable portable Oplette laser (410-2400 Opolette 355 LD, Opotek Inc. Carlsbad, CA, USA) was pulsed at $785 \mathrm{~nm}$ wavelength that was within the sensor excitation passband (600-1300 $\mathrm{nm}$ ) had incident on the SI head and transmitted through to the interrogating tissue. The repetition rate was set to the highest at $20 \mathrm{~Hz}$ with $7 \mathrm{~ns}$ FWHM pulse length. The laser was linearly polarized at $<2$ mrad divergence. The laser was connected to ICE450 pump laser power supply box for power and coolant circulation. The power supply box and laser were both controlled through the Opelette Control Box. The laser light was focused through a 10x magnification infinity-corrected microscope objective with a working distance of $10.6 \mathrm{~mm}$, numerical aperture (NA) of 0.25 and effective focal length (EFL) of $18 \mathrm{~mm}$. The focused laser beam with a 3-4 $\mathrm{mm}$ beam diameter was delivered through a $500 \mu \mathrm{m}$ core optical fiber $(\mathrm{NA}=0.22)$ to a silicone substrate 1 " diameter plano-convex spherical lens with a focal length from 15 $\mathrm{mm}-1000 \mathrm{~mm}$ (Thorlabs). The peak wavelength of the laser's spectral bandwidth can be tuned to multiple values for selective imaging of the different tissue compositions. The absorption of the laser energy produced acoustic pressure waves that propagated back to the SI head as autofluorescence signals and detected with the scanning peripheral system.

\section{Scanning System}

The SI scanning head read out these pressure waves by raster scanning with a $10 \mathrm{~mW}$ fibercoupled tunable CW external cavity laser (Thorlabs TLX1) operating at a nominal wavelength of 1550 $\mathrm{nm}$ focused interrogation laser beam over the SI surface. The beam was focused using a convex lens

onto the surface of the SI head at normal incidence angle to optically scan across the SI using an $x-y$ translatable optical scanner comprising of a pair of mutually orthogonal closed loop galvanometer mirrors. The reflected beam from the SI was directed via a polarizing beam splitter onto a $50 \mathrm{MHz}$ Indium Gallium Arsenide (InGaAs) photodiode (aperture $0.4 \mathrm{~mm}$ ) with transimpedance amplifier configuration of DC- and 
AC-coupled outputs. The DC-coupled output was connected to a $200 \mathrm{kS} / \mathrm{s}$ sampling rate, 16-bit resolution, 16-analog input channels universal multifunctional analog-to-digital (A-to-D, USB-4716, Advantech) card within the PC/DAQ and was record the transfer function (TF) as required for the phase bias control procedure. The AC-coupled output was connected to a $1 \mathrm{GHZ}$ bandwidth, 4 channels digitizing oscilloscope (DOS, InfiniiVision 6000 X-Series, Keysight Technologies) at 20 GS/s sampling rate to record the time-varying reflected optical power modulation produced by the incident fluorescence signal. The photodiode output was AC-coupled to remove the large DC optical component reflected from the SI and low-frequency fluctuations in the output power of the interrogation laser. ${ }^{1}$ The DOS acquired signals were downloaded to the PC/DAQ system. At each time point of the scan, the SI was optimally biased by tuning the interrogation laser wavelength to the point of maximum slope on the SI Transfer Function (TF) between the reflected optical power and phase (see Phase Bias Control for SI System). The entire system has fully automated with the excitation and interrogation lasers, the optical scanner, and the data acquisition hardware all under the control of a PC/DAQ.

\section{Auto-Fluorescence Signal Acquisition and Processing}

To acquire auto-fluorescence signal, the interrogation laser beam was first positioned at a random

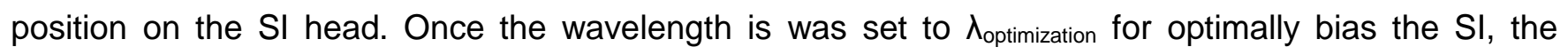
excitation tunable laser was then fired. At the same time, the DSO was triggered to begin acquiring the fluorescence signals. Once the acquisition was complete, the waveforms were downloaded to the PC and divided by the value of spatial point to compensate for sensitivity changes due to spatial variations in the reflectivity of the SI mirrors, defection of the Parylene film spacer, or fluctuations in the laser output power between acquisitions. The interrogation laser beam was moved to the next scan point and the process repeated until the scan was complete. Thus, for a 2D scan, the recorded data set was a 3D array $f(x, y, t)$, representing the incident time-varying fluorescence signal fover $x-y$ detection plane. The image reconstruction algorithm was based on an analytic inverse k-space method for planar and linear detection geometries with the premise that the spatial frequency components $f(x, y, z)$ were directly mapped on to 
it. Therefore, $f(x, y, z)$ can be recovered from the spatial and temporal frequency components of the set of detected fluorescence signals $f(x, y, t)$.

\section{Procedure of Histology}

After the imaging of CRi Maestro imaging of LCA and RCA, the samples were placed in $10 \%$ formalin to fix the tissue before submitting for plastic embedding and sectioning. Step sections $(5 \mu \mathrm{m}$ thick) were collected at different levels beginning at one end of the plaques and proceeding into the arterial lumen within the embedded device. The sections were mounted on glass microscopic slides, stained with Verhoeff's Van Gieson (EVG) and Haemotoxylin and Eosin (H\&E) reagents and covered with glass cover slips. The microscopic slides were examined with an Olympus BX43 microscope fitted with Olympus UC90 Digital camera for diffuse white light (LM) and transmission polarizing (TPM) optics and $2 \times, 20 \times$, and 100x objectives. Selected fields were imaged using Zeiss Axiocam MRc5 digital camera mounted on the microscope and Zeiss AxioVision SE64 Rel 4.8 software supported by a Dell Optiplex 980 Desktop Computer. Selected images were further modified using Adobe Photoshop Elements 9 software.

\section{Region-of-Interest (ROI) Calculation}

Autofluorescence signals from both in vivo and ex vivo LCA images were calculated based on the selected ROls (same size for all samples). An average of mean signal intensity was then calculated in arbitrary units (A.U.) for the SI images. An average radiant efficiency was calculated with a unit of $\left(\mathrm{p} / \mathrm{sec} / \mathrm{cm}^{2} / \mathrm{sr}\right) /\left(\mu \mathrm{W} / \mathrm{cm}^{2}\right)$ based on the Maestro images.

\section{Statistical Analysis}

Linear Regression analyses of the autofluorescence signals from IPH and neovascularization were performed to investigate any correlation between the results collected with SI and CRi imaging systems. Also, QQ plots were analyzed to identify the underlying distribution of autofluorescence signal 
from IPH and neovascularization, two of the natural endogenous fluorophores detected with our SI imaging system as well as the CRi Maeastro fluorescence imaging system. An ANOVA was performed to compare in vivo and ex vivo signal intensity from ligated LCAs at various exposure time. Mice weight and dose were not considered in the statistical analysis due similarity among the mice. We used $\mathrm{P}<0.05$ for statistical significance for all in vivo and ex vivo signal analyses

Statistical analysis clearly showed, $2700 \times\left(\right.$ LCA: $1.71 \times 10^{7}$ vs. RCA: $\left.6.33 \times 10^{3}\right)$ higher autofluorescence signal from atherosclerotic plaques in LCA due to IPH with the SI imaging system, which is similar to $2580 \times\left(\right.$ LCA: $1.63 \times 10^{7}$ vs. RCA: $\left.6.32 \times 10^{3}\right)$ for the CRi Maestro imaging. Neovascularization exhibited $2920 \times\left(\right.$ LCA: $1.73 \times 10^{7}$ vs. RCA: $5.92 \times 10^{3}$ ) brighter autofluorescence from neovascularization with the SI imaging system that is similar to $2910 \times\left(\right.$ LCA: $1.72 \times 10^{7}$ vs. RCA: $\left.5.90 \times 10^{3}\right)$ for CRi Maestro imaging (Fig. S1). Linear regression models show a very close correlation between the collected autofluorescence signals with SI and CRi imaging systems from intraplaque hemorrhage $\left(R^{2}=0.99236\right.$, Fig. S2a) and neovascularization $\left(R^{2}=0.9699\right.$, Fig. S2b). $Q Q$ plots show normal distribution of autofluorescence signal due to intraplaque hemorrhage and neovascularization regardless of SI and CRi Maestro imaging systems (Figs. S2c-f). All detected autofluorescence signals match with the theoretical quantiles distribution. This proofs our assumption that autofluorescence signal from both IPH and neovascularization has a linear relationship with the exposure time of the tissue to laser source. Based on Student t-test it was identified that autofluorescence signal at each exposure time are statistically significant (Fig. S3a). However, signal intensity for no exposure is not significant difference with 5 seconds exposure but significant difference in exposure for 10-25 seconds (Fig. S3b). The autofluorescence signal for exposure for 5 seconds is significantly different to exposures $10-25$ seconds and not significant difference with no exposure (Fig. S3c), on the other hand, exposure for 10 seconds is significantly different than no exposure, 5, 15, 20, and 25 minutes exposure (Fig. S3d). Signal intensity for 15 seconds exposure is only significant with 20 seconds exposure (Fig. S3e). Autofluorescence signal for exposure 20 seconds is not significantly difference with exposures 15 and 25 seconds (Fig. S3f), on 
the other hand exposure 25 second is significantly different with $0-15$ seconds exposure but not significant difference with 20 seconds (Fig. S3g).

\section{SUPPLEMENTAL FIGURES}

Figure S1. Statistical analyses of autofluorescence signal in mice carotid atherosclerotic plaques. (a) Average autofluorescence signal from in vivo mice carotid atherosclerotic plaques due to IPH that is verified with (b) ex vivo CRi Maestro fluorescence imaging system with the same samples after euthanizing the animals. (c) Average autofluorescence signal from in vivo mice carotid atherosclerotic plaques due to neovascularization that is verified with (d) ex vivo CRi Maestro fluorescence imaging of the same samples after euthanizing the animals. These results are statistically significant $(P<0.05)$.

Figure S2. Linear regression models show a very close correlation between the collected autofluorescence signals with $\mathrm{SI}$ and $\mathrm{CRi}$ imaging systems from (a) intraplaque hemorrhage $\left(\mathrm{R}^{2}=0.99236\right)$ and (b) neovascularization $\left(R^{2}=0.9699\right)$. (c-f) $Q Q$ plots show a normal distribution of autofluorescence generated from intraplaque hemorrhage and neovascularization regardless of SI and CRi Maestro imaging systems.

Figure S3. Student ANOVA t-test to compare autofluorescence signal at all 6 exposure time 0, 5, 10, 15, 20, 25 seconds. (a) All results are statistically significant. Mean autofluorescence signal at (b) 0 second (no laser exposure) exposure is significantly different than 10-25 seconds exposure, but not with 5 seconds exposure; (c) 5 seconds exposure is significantly different than 10-25 seconds exposure, but not with 0 second exposure; (d) 10 seconds exposure is significantly different than $0,5,15-25$ seconds exposure; (e) 15 seconds exposure is significantly different than $0-10,25$ seconds exposure, but not with 20 seconds exposure; (f) 20 seconds exposure is significantly different than 0-10 seconds exposure, but not with 15 and 25 seconds exposure; (g) 25 seconds exposure is different than 0-15 seconds exposure, but not with 20 seconds exposure. 


\section{SUPPLEMENTAL RESULTS}

(a)

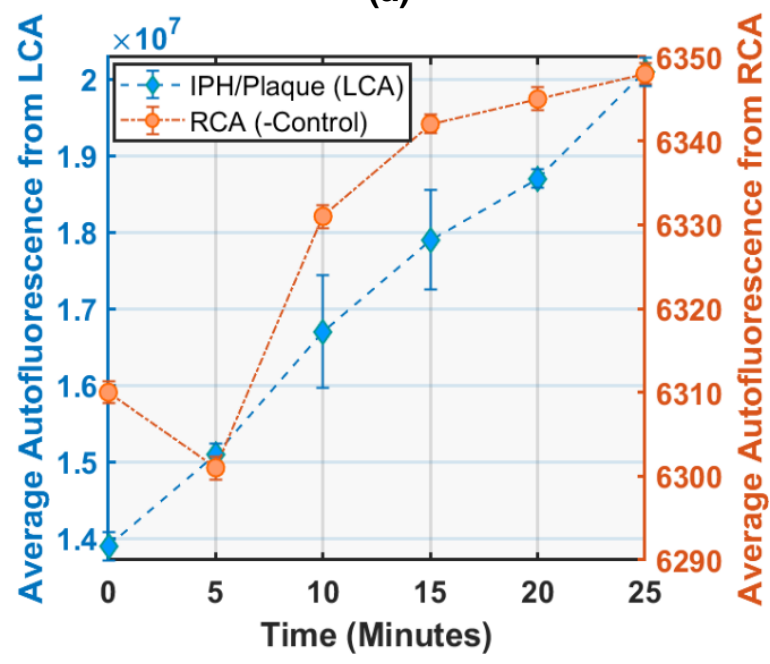

(c)

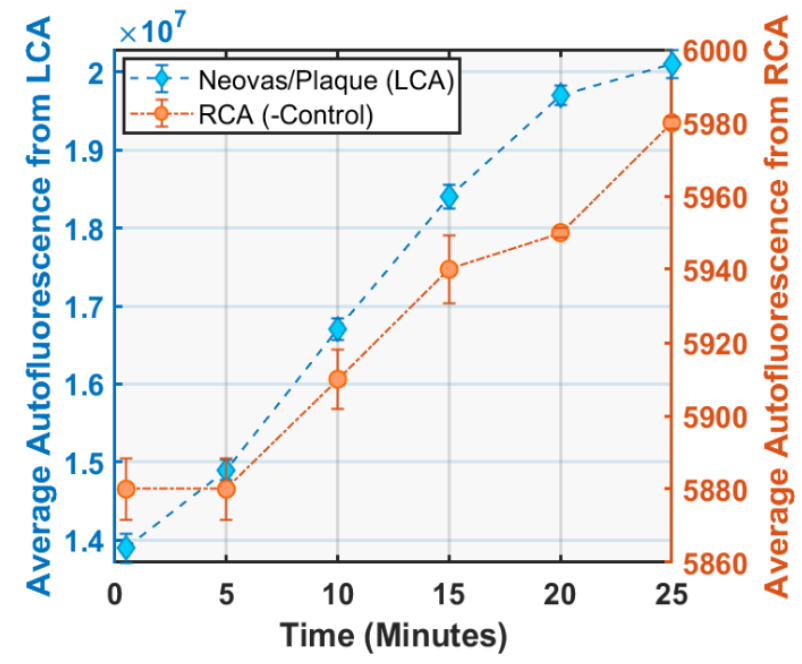

(b)

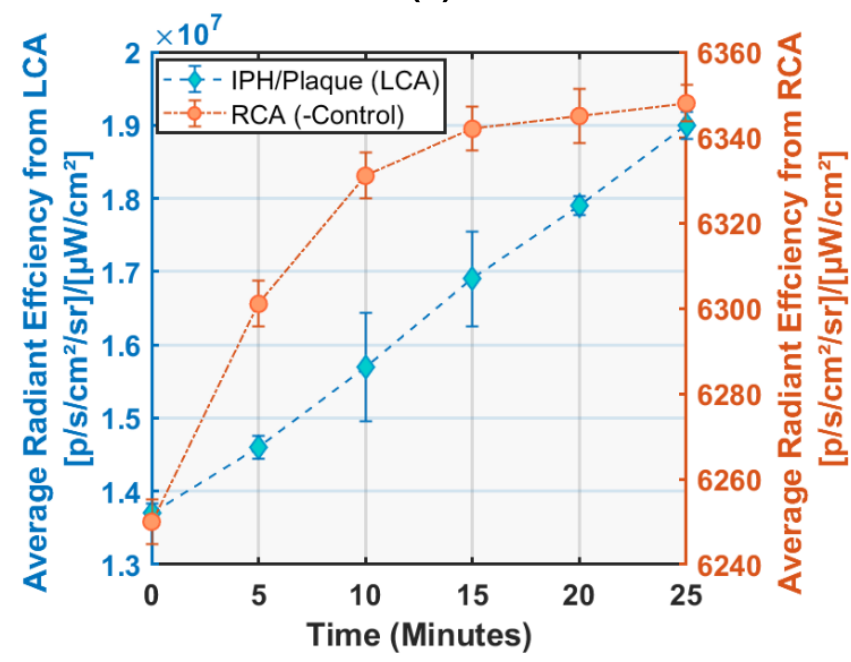

(d)

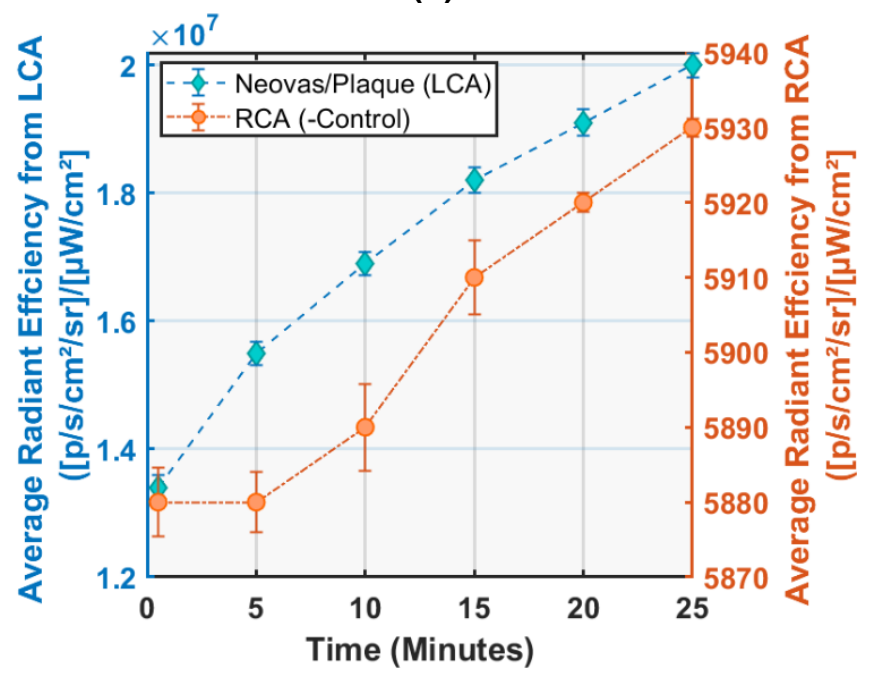

Figure S1. Statistical analyses of autofluorescence signal in mice carotid atherosclerotic plaques. (a) Average autofluorescence signal from in vivo mice carotid atherosclerotic plaques due to IPH that is verified with (b) ex vivo $\mathrm{CRi}$ Maestro fluorescence imaging system with the same samples after euthanizing the animals. (c) Average autofluorescence signal from in vivo mice carotid atherosclerotic plaques due to neovascularization that is verified with (d) ex vivo CRi Maestro fluorescence imaging of the same samples after euthanizing the animals. These results are statistically significant $(P<0.05)$. 
(a)

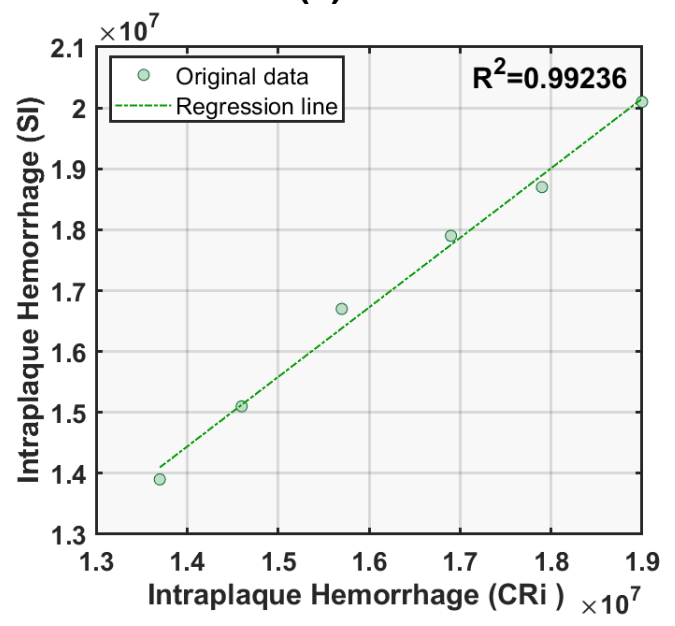

(c)

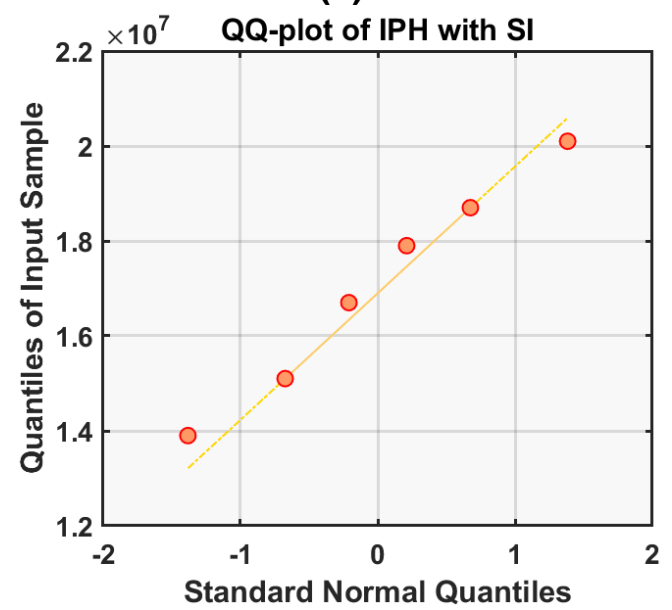

(e)

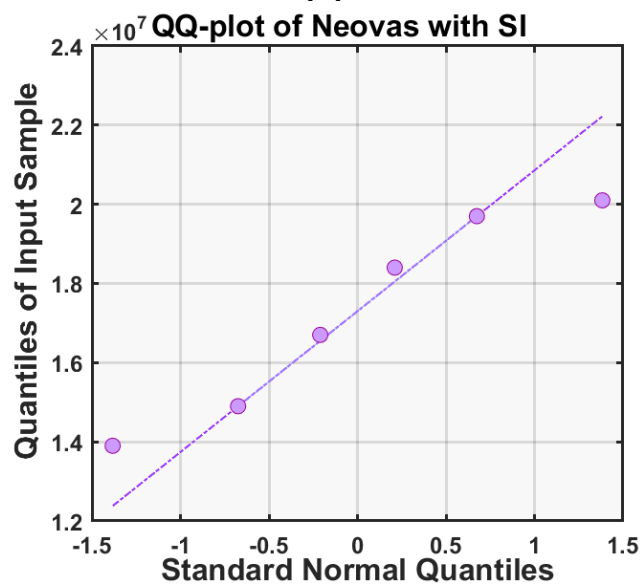

(b)

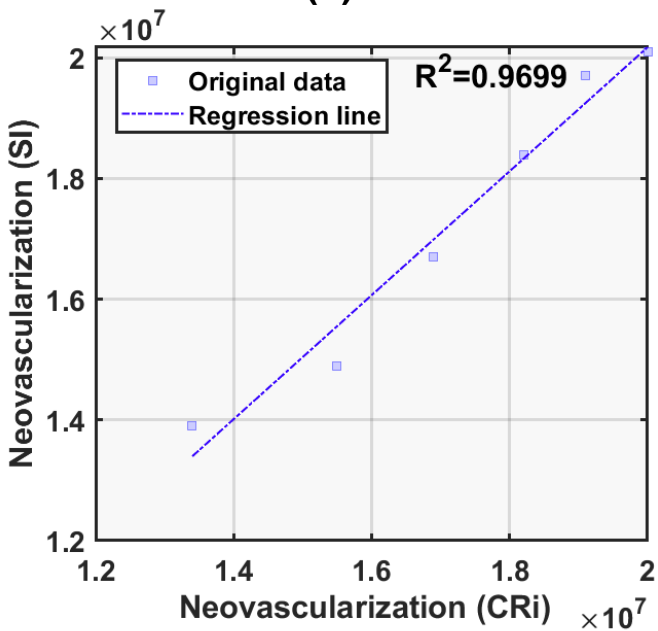

(d)

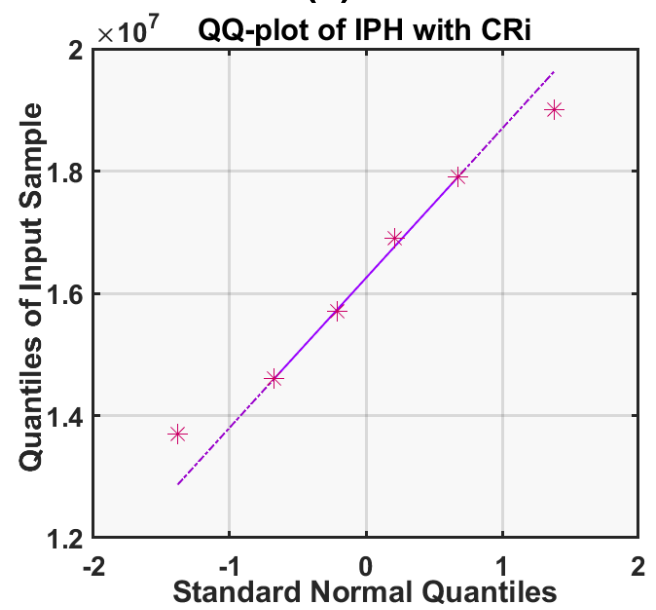

(f)

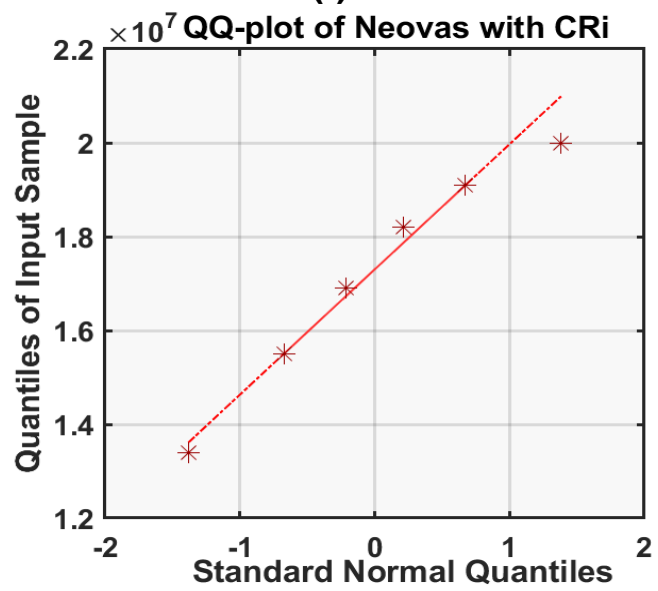

Figure S2. Linear regression models show a very close correlation between the collected autofluorescence signals with $\mathrm{SI}$ and $\mathrm{CRi}$ imaging systems from (a) intraplaque hemorrhage $\left(R^{2}=0.99236\right)$ and (b) neovascularization $\left(R^{2}=0.9699\right)$. (c-f) $Q Q$ plots show a normal distribution of autofluorescence generated from intraplaque hemorrhage and neovascularization regardless of SI and CRi Maestro imaging systems. 


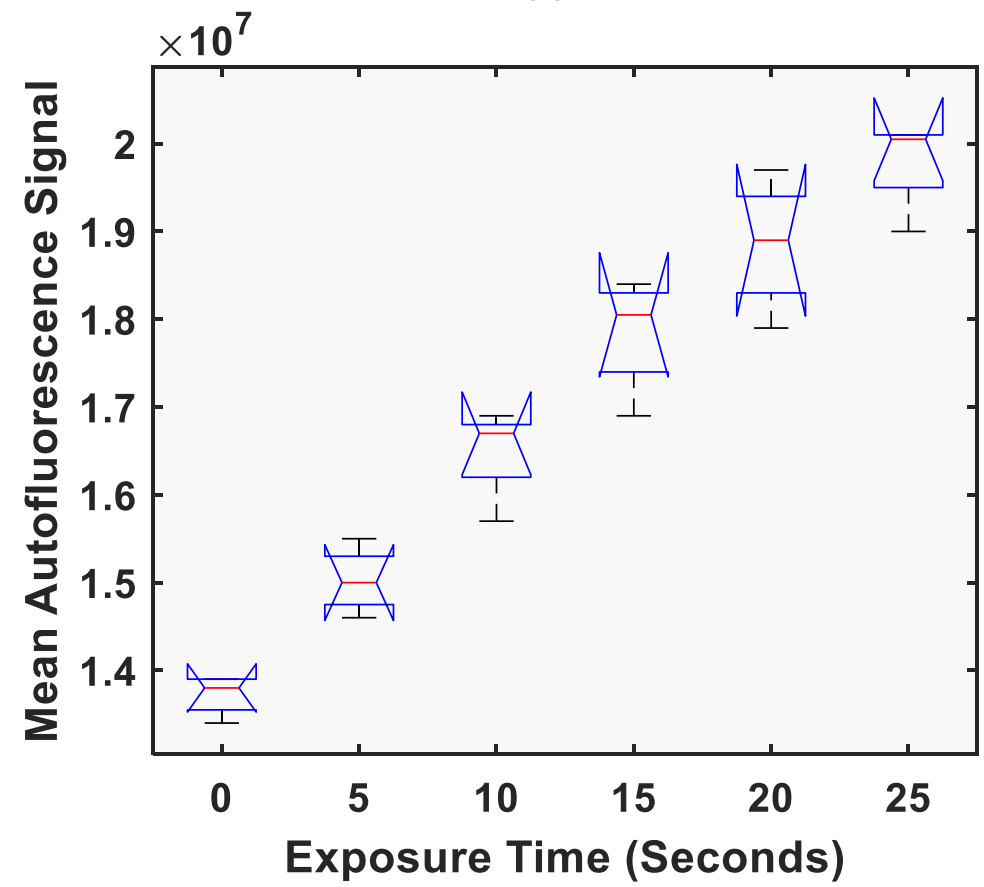

(b)

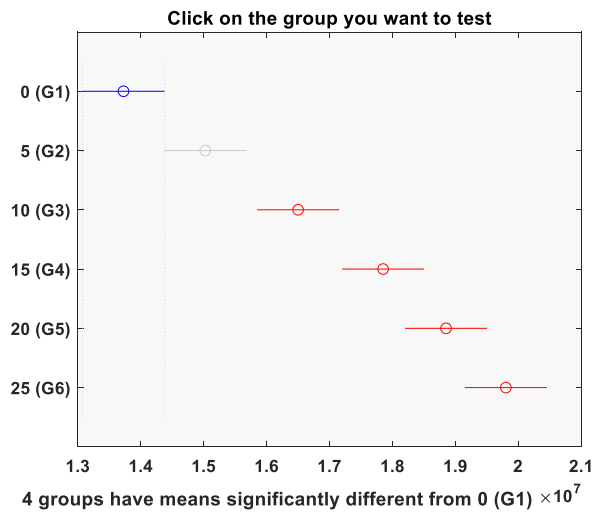

(e)

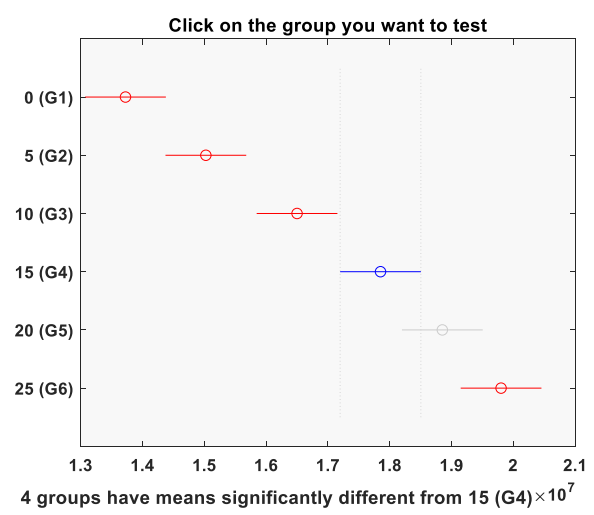

(c)

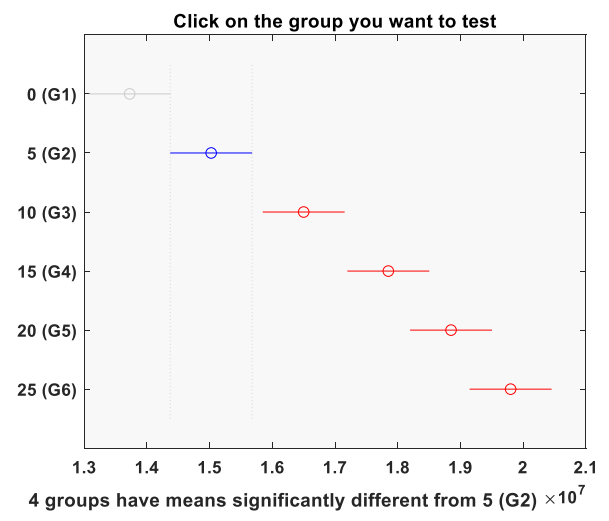

(f)

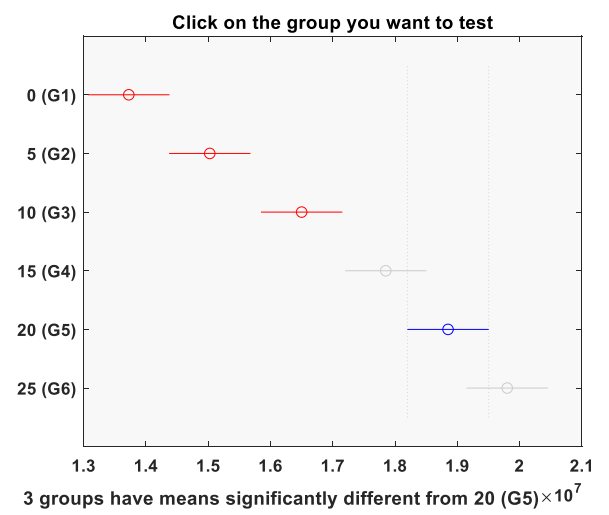

(d)

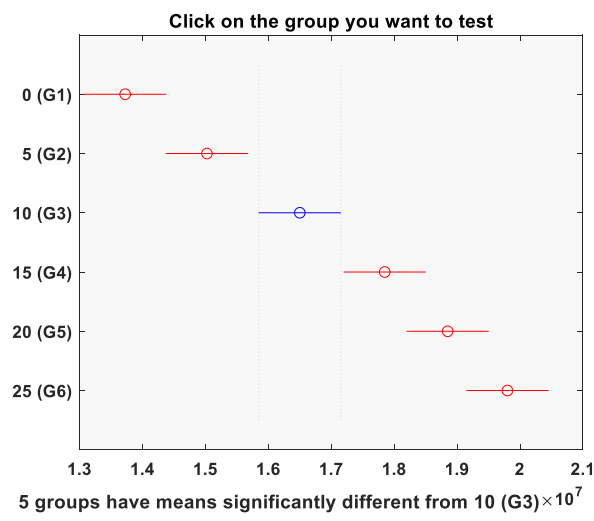

(g)

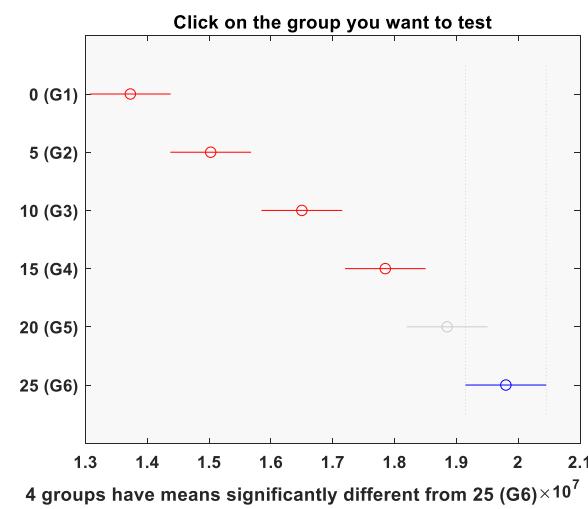

Figure S3. Student ANOVA t-test to compare autofluorescence signal at all 6 exposure time 0,5, 10, 15, 20, and 25 seconds represent 6 Groups: G1, G2, G3, G4, G5, and G6 respectively. (a) All results are statistically significant. Mean autofluorescence signal at (b) 0 second (no laser exposure) exposure is significantly different than 10-25 seconds exposure, but not with 5 seconds exposure; (c) 5 seconds exposure is significantly different than 10-25 seconds exposure, but not with 0 second exposure; (d) 10 seconds exposure is significantly different than 0, 5, 15-25 seconds exposure; (e) 15 seconds exposure is significantly different than $0-10,25$ seconds exposure, but not with 20 seconds exposure; (f) 20 seconds exposure is significantly different than $0-10$ seconds exposure, but not with 15 and 25 seconds exposure; (g) 25 seconds exposure is different than $0-15$ seconds exposure, but not with 20 seconds exposure. 


\section{REFERENCES}

1. Beard, P. C., Interrogation of free-space Fabry-Perot sensing interferometers by angle tuning. Measurement Science and Technology 2003, 14 (11), 4. 